\title{
Intra-articular triamcinolone hexacetonide in knee osteoarthritis: factors influencing the clinical
} response

\author{
K Gaffney, J Ledingham, J D Perry
}

\begin{abstract}
Objective-To assess the efficacy of a single intra-articular injection of triamcinolone hexacetonide (THA) in knee osteoarthritis (OA) and examine factors which may relate to treatment efficacy. Methods-Eighty four patients with clinical and radiographic evidence of knee OA were recruited and randomly allocated to receive either THA (20 mg in $1 \mathrm{ml})$ or placebo $(0.9 \%$ normal saline, $1 \mathrm{ml})$. Follow up assessments evaluated the following outcome variables: patient opinion of overall change in the treated knee, visual analogue pain score (VAS), distance walked in one minute (WD), and Health Assessment Questionnaire modified for lower limb function (HAQ).
\end{abstract}

Results-Seventy eight percent of THA and $49 \%$ of placebo treated patients reported overall improvement at week 1 $(\mathrm{p}<0 \cdot 05)$. At week 6, improvement was reported in $57 \%$ and $55 \%$ of patient groups, respectively. VAS improved in both groups at week 1 (THA, p < 0.001; placebo, $p<0.05$ ) and week 6 (both $p<0.01)$. Improvement in VAS was significantly greater among THA treated patients at week 1 only $(p<0.01)$. Subgroup analysis of THA treated patients revealed greater improvment in VAS among patients with clinical evidence of an effusion $(p<0.05)$, and those who had synovial fluid successfully aspirated at the time of injection $(p<0.01)$. WD improved in THA treated patients at week 1 $(p<0.001)$, and in both groups at week 6 (THA, p<0.001; placebo, p<0.01). Improvements in HAQ were seen in THA patients only at weeks 1 and $6(p<0.05)$. Regression analysis did not identify any additional clinical, radiographic, or synovial fluid characteristics which influenced the response.

Conclusions-THA provided short term pain relief in knee $O A$. Increased benefit was associated with both clinical evidence of joint effusion and successful aspiration of synovial fluid at the time of injection.

(Ann Rheum Dis 1995; 54: 379-381)

Osteoarthritis (OA) of the knee is one of the most common rheumatic disorders and a frequent cause of pain and disability, particularly among the elderly. ${ }^{12}$ It is estimated that between 18 and $33 \%$ of individuals over 65 years of age have radiographic evidence of knee $\mathrm{OA}$, this being more common in women and increasing to over $50 \%$ by age $80 .{ }^{3}$ Between 20 and $60 \%$ of patients with radiographic disease have associated symptoms, ${ }^{45}$ and up to $50 \%$ report disability. ${ }^{6}$ Despite the enormous public health problem presented by this condition, there is no singularly effective medical treatment. Two double blind placebo controlled studies have reported a transient benefit from intra-articular triamcinolone hexacetonide (THA), ${ }^{78}$ although patient numbers were small and clinical responses variable. In view of concern regarding efficacy and possible deleterious effects, judicious use of intraarticular steroids in OA has been advised. ${ }^{7}$

Studies in experimental models of OA have shown that low dose steroids reduce the size, severity, and progression of both cartilage lesions and osteophyte formation, ${ }^{9-11}$ although previous reports have raised the possibility that steroids may have adverse effects on articular cartilage..$^{12}$ In view of these conflicting data, any reported clinical benefit in knee $O A$ assumes greater significance if subgroups of 'responders' and 'non-responders' can be identified in order to facilitate patient preselection. We conducted this placebo controlled study in an attempt to re-evaluate the efficacy of a single intra-articular injection of THA in knee OA and examine factors which may relate to treatment efficacy.

\section{Patients and methods}

After receiving ethics committee approval, we invited patients with clinical and radiographic evidence of knee $\mathrm{OA}$ who presented to a general rheumatology clinic with knee pain and functional impairment (modified Health Assessment Questionnaire $>0$ ) during a six month period to participate in the study. In those patients with bilateral knee OA, the most painful knee was studied. Patients were randomly allocated to receive either THA $(20 \mathrm{mg}$ in $1 \mathrm{ml}$ ) or placebo (1 $\mathrm{ml}$ of $0.9 \%$ normal saline). Informed written consent was obtained from all participants.

At the time of recruitment, rheumatological history and examination were undertaken and the patient's age, sex, height, weight, history and outcome of previous intra-articular corticosteroid injections, pattern of joint involvement, and duration of knee symptoms recorded. Knee pain experienced during the previous week (expressed on a $100 \mathrm{~mm}$ horizontal visual analogue pain scale (VAS)), 
distance walked in one minute (WD) and Health Assessement Questionnaire modified for lower limb function (HAQ) ${ }^{14}$ were also measured. Clinical evidence of joint effusion (positive bulge sign and patellar tap: present/ absent) was documented. Knee aspiration was performed via the medial approach and the volume of aspirated synovial fluid (SF) recorded. Total and differential leucocyte count were estimated in all SF samples, which were also examined for the presence of calcium pyrophosphate crystals by one experienced observer (JL) utilising polarising light microscopy. Following aspiration, knees were randomly injected with THA $(20 \mathrm{mg}$ in $1 \mathrm{ml})$ or placebo ( $1 \mathrm{ml}$ of $0.9 \%$ normal saline).

Standard weight bearing anteroposterior and lateral knee radiographs were obtained. Each compartment (medial tibiofemoral, lateral tibiofemoral, patellofemoral) was graded $0-3$ for overall severity of OA. ${ }^{15}$ In addition, individual features in each compartment (narrowing, sclerosis, osteophytes, cysts, and attrition) were graded $0-3$ and presence/ absence of chondrocalcinosis noted. ${ }^{15}$ Clinical assessments were performed by one blinded clinician (KG); joint aspiration, injection, radiological scoring, and SF crystal analysis were undertaken by another $(\mathrm{JL})$.

At follow up visits (weeks 1 and 6), patient opinion of overall change in the treated knee (worse, no change, improved), pain score (VAS), WD, and HAQ were recorded. At completion of the study, patients who received placebo were offered THA therapy.

\section{STATISTICAL ANALYSIS}

Changes in VAS, WD, and HAQ at weeks 1 and 6 were compared with baseline using Student's paired $t$ test. Analysis of variance for repeated measures was used to compare differences in THA, placebo, and subgroups (effusion present/absent, synovial fluid aspirated yes/no), respectively. In THA treated patients, the relationship between improvement in VAS at week 1 and other clinical variables (patient age, duration of symptoms, $\mathrm{HAQ}$, radiographic severity score, SF volume, SF leucocyte count) was assessed by simple regression analysis. The differences in patient opinion of overall change, and relationship

Table 1 Patient demographic data

\begin{tabular}{lcc}
\hline & Triamcinolone & Placebo \\
\hline Age (yr) & $66(9 \cdot 7)$ & $68(8 \cdot 6)$ \\
Sex $(\mathrm{M} / \mathrm{F})$ & $9 / 33$ & $15 / 27$ \\
Height $(\mathrm{m})$ & $1 \cdot 6(0 \cdot 08)$ & $1 \cdot 62(0 \cdot 09)$ \\
Weight $(\mathrm{kg})$ & $75(15 \cdot 1)$ & $79(15 \cdot 1)$ \\
Symptom duration (yr) & $6 \cdot 7(6 \cdot 6)$ & $7 \cdot 1(6 \cdot 3)$ \\
Disease pattern & 18 & 22 \\
$\quad$ Knee & 24 & 20 \\
Generalised & $4 \cdot 7(2 \cdot 1)$ & $3 \cdot 7(1 \cdot 6)$ \\
Modified HAQ (0-9) & 10 & 13 \\
Previous intra-articular steroid & $(90)$ & $(90)$ \\
$\quad \%$ reporting improvement) & $3 \cdot 5(1 \cdot 8)$ & $3 \cdot 7(1 \cdot 6)$ \\
Radiological score (0-9) & 4 & 12 \\
$\quad$ Chondrocalcinosis & 17 & 16 \\
Clinical effusion & 24 & 26 \\
Synovial fluid aspirated & 2 & 6 \\
Pyrophosphate crystals & $1 \cdot 0(0 \cdot 76)$ & $0 \cdot 85(0 \cdot 47)$ \\
SF total leucocyte count $\left(\times 10^{9} / 1\right)$ &
\end{tabular}

Results expressed as mean (SD) or actual patient numbers. between clinical evidence of joint effusion and successful SF aspiration were calculated by contingency table analysis. Differences which were significant at the $5 \%$ confidence interval are quoted.

\section{Results}

Patient demographic data were similar in both patient groups (table 1). Thirty eight percent of patients had clinical evidence of joint effusion; however, SF was aspirated from $60 \%$ of the total population (median volume $5 \mathrm{ml}$, range $0-85$ ), 33\% having greater than $5 \mathrm{ml}$. Clinical evidence of joint effusion correlated with successful aspiration of SF ( $p<0.01)$.

Seventy eight percent of THA and $49 \%$ of placebo treated patients reported overall improvement at week $1(\mathrm{p}<0.05)$. At week 6 , improvement was reported in $57 \%$ and $55 \%$ of THA and placebo treated groups, respectively. Four placebo treated patients reported a deterioration at week 1 ; two of these withdrew from the study because of lack of treatment efficacy. At week 1, subgroup analysis of THA treated patients revealed that $76 \%$ of those with clinical evidence of joint effusion (compared with $80 \%$ without effusion) reported overall benefit; among patients who had SF successfully aspirated at the time of injection, $92 \%$ reported benefit (compared with $61 \%$ without SF; $p<0.05$ ). At week 6 , overall benefit was reported by $53 \%$ of THA treated patients with a joint effusion (compared with $60 \%$ without effusion), and $66 \%$ of patients who had SF successfully aspirated (compared with $44 \%$ without $S F$ ).

Table 2 outlines changes in VAS, WD, and HAQ in THA and placebo treated patients at weeks 0,1 , and 6 . Both groups demonstrated a significant improvement in VAS at week 1 (THA, p < 0.001; placebo, p < 0.05) and week 6 (both $\mathrm{p}<0.01$ ). WD improved in THA treated patients only at week $1(p<0.001)$, and in both groups at week 6 (THA, p < 0.001; placebo, $p<0.01)$. Improvements in HAQ were seen in THA patients only at weeks 1 and $6(\mathrm{p}<0.05)$. At week 1 , improvement in VAS was greatest in THA treated patients $(\mathrm{p}<0.01)$.

Sub-group analysis of THA treated patients revealed that improvement in VAS was greater among patients with clinical evidence of joint effusion $(p<0.05)$ and those who had SF successfully aspirated at the time of injection $(p<0.01)$ (table 3). Aspiration of SF (compared with inability to aspirate SF) was

Table 2 VAS, WD and $H A Q$ in triamcinolone and placebo groups

\begin{tabular}{|c|c|c|c|}
\hline & Week 0 & Week 1 & Week 6 \\
\hline $\begin{array}{c}\text { VAS (mm) } \\
\text { THA } \\
\text { Placebo }\end{array}$ & $\begin{array}{l}52 \cdot 0(21 \cdot 1) \\
57 \cdot 0(22 \cdot 0)\end{array}$ & $\begin{array}{l}21 \cdot 7(20 \cdot 7)^{\star \star \star}+十 \\
43 \cdot 1(28 \cdot 7)^{\star}\end{array}$ & $\begin{array}{l}35.8(26.8)^{\star \star} \\
42.9(26.0)^{\star \star}\end{array}$ \\
\hline $\begin{array}{c}\text { WD (m) } \\
\text { THA } \\
\text { Placebo }\end{array}$ & $\begin{array}{l}48.9(15.5) \\
46.5(15.9)\end{array}$ & $\begin{array}{l}50 \cdot 7(15 \cdot 3)^{\star \star \star} \\
48 \cdot 3(16 \cdot 3)\end{array}$ & $\begin{array}{l}50 \cdot 8(15 \cdot 4)^{\star \star \star} \\
51.6(14 \cdot 5)^{\star \star}\end{array}$ \\
\hline $\begin{array}{l}\text { HAQ } \\
\text { THA } \\
\text { Placebo }\end{array}$ & $\begin{array}{l}4 \cdot 7(2 \cdot 1) \\
4 \cdot 4(1 \cdot 9)\end{array}$ & $\begin{array}{l}4 \cdot 2(2 \cdot 1)^{\star} \\
4 \cdot 3(1 \cdot 9)\end{array}$ & $\begin{array}{l}4 \cdot 5(2 \cdot 3)^{\star} \\
4 \cdot 2(2 \cdot 0)\end{array}$ \\
\hline
\end{tabular}

$\star \star \star \mathrm{p}=0.001$, Student's $t$ test; $t+\mathrm{p}<0.01$, ANOVA. 
Table 3 VAS in triamcinolone treated subgroups

\begin{tabular}{clll}
\hline & Week 0 & Week 1 & Week 6 \\
\hline Joint effusion & & & \\
Yes (n=17) & $43.5(21 \cdot 8)$ & $14 \cdot 6(11 \cdot 8)^{\star \star} \dagger$ & $32 \cdot 0(26 \cdot 2)^{\star}$ \\
No (n=25) & $57.8(26 \cdot 7)$ & $26.5(21 \cdot 1)^{\star \star}$ & $38.4(32 \cdot 1)^{\star}$ \\
SF aspirated & & & \\
Yes (n=24) & $48.5(19 \cdot 1)$ & $11 \cdot 1(10 \cdot 6)^{\star \star \star}+\dagger$ & $35 \cdot 1(27 \cdot 6)^{\star}$ \\
No $(\mathrm{n}=18)$ & $56.6(23 \cdot 2)$ & $35.9(22 \cdot 7)^{\star \star}$ & $36 \cdot 7(26 \cdot 4)^{\star \star}$ \\
\hline
\end{tabular}

Results expressed as means (SD). ${ }^{\star} p<0.05,{ }^{\star \star} p<0.01$ ${ }_{\star \star \star} \mathrm{p}=0.001$, Student's $t$ test; $\mathrm{tp}<0.05, \mathrm{ttp}<0.01$, ANOVA.

not associated with greater reduction in VAS in the placebo group. In the THA group, simple regression analysis did not reveal any association between improvement in VAS and any of the following: duration of symptoms, baseline VAS, WD, or HAQ, radiographic score, SF volume, or SF leucocyte count.

\section{Discussion}

The heterogeneity of OA has been increasingly recognised and a variety of patient subgroups with an underlying inflammatory/autoimmune aetiology proposed. Intra-articular THA is often used as a therapeutic strategy in the management of the conventional inflammatory arthropathies, but has not reached a similar level of clinical acceptance in OA. In knee OA, anecdotal experience suggests that there is a tendency to reserve this form of therapy for patients with significant disability and advanced radiographic disease who have not responded to more conventional treatment. This clinical practice may change as a result of recent experimental evidence which suggests that intra-articular steroids exert a chondroprotective effect, possibly mediated by suppression of synthesis of stromelysin, ${ }^{9}$ a metalloprotease implicated in $\mathrm{OA}$ cartilage degradation.

We have demonstrated that intra-articular THA provides significant short term pain relief in knee OA. Additional benefit was observed both among patients with clinical evidence of a joint effusion, and in those who had SF successfully aspirated at the time of injection, the magnitude of the response being greater in the latter group. Although this study was not, by strict definition, double-blinded, we attempted to ensure that patients were not aware of the treatment allocated to them, by shielding the identity of the treatment received from their view at the time of injection; only the injecting physician (JL) was aware of the nature of the injection administered.

It has been suggested that variations in the response to intra-articular steroid therapy may be attributable to inaccurate injection. ${ }^{16}$ In that study, Jones et al addressed the issue by obtaining a single plain joint radiograph after joint aspiration and injection in patients who received methyl prednisolone mixed with a radiographic contrast medium. The study addressed accuracy of injection in a series of joints including 59 knees from 109 patients with a spectrum of rheumatological conditions (52 osteoarthritis). Accurate injection was associated with successful aspiration of SF at the time of injection. Reduction in joint inflammation was, in turn, associated with accurate injection. Thirty four percent of knee injections alone were extra-articular or uncertain in this study. These observations suggest that the association between pain relief and successful aspiration of SF demonstrated in our study may relate to accuracy of injection rather than the fact that these patients represent a specific 'inflammatory' knee OA subgroup.

Previous studies have suggested that OA patients with crystal releated synovitis benefit from intra-articular steroid therapy. ${ }^{17}$ This could not be reliably assessed in our study, as only small numbers of patients with either chondrocalcinosis $(n=16)$ or pyrophosphate crystals $(n=8)$ were identified, the majority of whom were randomly allocated to receive placebo.

We suggest that a trial of intra-articular THA therapy should be considered in knee OA, and may be a useful adjunct to other therapies. This is particularly true of the elderly population, in whom knee $\mathrm{OA}$ is a source of considerable pain and disability and therapeutic options are often extremely limited. Any additional possible long term chondroprotective effect must be evaluated further.

1 Hadler N M. Osteoarthritis as a public health problem. Clin Rheum Dis 1985; 11: 175-85.

2 Wood P N H. Osteoarthritis in the community. Clin Rheum Dis 1976; 2: 495-507.

3 Van Saase J L C M, Van Romunde L K S, Cats A, Vandenbroucke J P, Valkenburg H A. Epidemiology of osteoarthritis: Zoetermeer survey. Comparison of radiological osteoarthritis in a Dutch population with that in 10 other populations. Ann Rheum Dis 1989; 48: 271-80.

4 Felson D T, Naimark A, Anderson J, Kazis J, Castelli W, Meenan R P. The prevalence of knee osteoarthritis in the elderly: the Framingham osteoarthritis study. Arthritis Rheum 1987; 30: 914-8.

5 Hart D J, Spector T D, Brown P, Wilson P, Doyle D V, Silman A J. Clinical signs of early osteoarthritis: reproducibility and $x$-ray changes in 541 women in the general population. Ann Rheum Dis 1991; 50: the general

6 Davis M A, Ettinger W H, Neuhaus J M, Mallon K P. Knee $O A$ and physical functioning: evidence from the NHANES epidemiological follow-up study. $f$ Rheumatol 1991; 18: 591-8.

7 Friedman D M, Moore M E. The efficacy of intra-articular steroids in OA; a double blind study. $\mathcal{F}$ Rheumatol 1980; 7: 850-6.

8 Dieppe P A, Sathapatayavongs B, Jones H E, Bacon P A, Ring E F J. Intra-articular steroids in osteoarthritis. Rheumatol Rehabil 1980; 19: 212-7.

9 Pelletier J-P, Mineau F, Raynauld J-P, Woessner J F, GunjaSmith Z, Martel-Pelletier J. Intraarticular injections with methylprednisolone acetate reduce osteoarthritic lesions in parallel with chondrocyte stromelysin synthesis in in parallel with chondrocyte stromelysin synthesis in 414-23.

10 Pelletier J-P, Martel-Pelletier J. Protective effects of corticosteroids on cartilage lesions and osteophyte formation in the Pond-Nuki dog model of osteoarthritis. Arthritis Rheum 1989; 32: 181-93.

11 Williams J M, Brandt K D. Triamcinolone hexacetonide protects against fibrillation and osteophyte formation following chemically induced articular cartilage damage. Arthritis Rheum 1985; 28: 1267-74.

12 Behrens, F, Shepard N, Mitchell N. Alterations of rabbit articular cartilage by intra-articular injections of articular cartilage by intra-articular injections

13 Moskowitz R W, Davis W, Sammarco J, Mast W, Chase S W. Experimentally induced corticosteroid Chase S W. Experimentally induced cortico

14 McAlindon T E, Cooper C, Kirwan J R, Dieppe P. Determinants of disability in osteoarthritis of the knee. Ann Rheum Dis 1993; 52: 258-62.

15 Ledingham J, Regan M, Jones A, Doherty M. Radiographic patterns and associations of osteoarthritis of the knee in patients referred to hospital. Ann Rheum Dis 1993; 52: 520-6.

16 Jones A, Regan M, Ledingham J, Pattrick M, Manhire A, Doherty $M$. Importance of placement of intra-articular steroid injections. $B M F$ 1 1993; 307: 1329-30.

17 Dieppe P A. Are intra-articular steroid injections useful for the treatment of the osteoarthritis joint? Br $\mathcal{F}$ Rheumatol 1991; 30: 199. 